\title{
Upper Bounds on the Diameter of Bipartite and Triangle-Free Graphs with Prescribed Edge Connectivity
}

\author{
Blessings T. Fundikwa $\mathbb{D}^{1},{ }^{1}$ Jaya P. Mazorodze, ${ }^{1}$ and Simon Mukwembi ${ }^{2}$ \\ ${ }^{1}$ Department of Mathematics, University of Zimbabwe, Harare, Zimbabwe \\ ${ }^{2}$ School of Mathematics, Statistics and Computer Science, University of KwaZulu-Natal, Durban, South Africa \\ Correspondence should be addressed to Blessings T. Fundikwa; takudzwabf@gmail.com
}

Received 30 May 2020; Revised 14 July 2020; Accepted 22 July 2020; Published 3 September 2020

Academic Editor: N. Hussain

Copyright (c) 2020 Blessings T. Fundikwa et al. This is an open access article distributed under the Creative Commons Attribution License, which permits unrestricted use, distribution, and reproduction in any medium, provided the original work is properly cited.

We present upper bounds on the diameter of bipartite and triangle-free graphs with prescribed edge connectivity with respect to order and size. All bounds presented in this paper are asymptotically sharp.

\section{Introduction}

Graph theory is used to study the mathematical structures of pairwise relations among objects. Mathematically, a pair $G=$ $(V, E)$ is a crisp graph, where $V$ is a nonempty set and $E$ is a relation on $V$ [1]. The order of a graph $G$ is the number of vertices of $G$ and is denoted by $|V|=n$. The size of $G$, denoted by $|E|=m$, is the number of edges of $G$. The distance, $d_{G}(u, v)$, between two vertices $u, v$ of $G$ is the length of a shortest $u-v$ path in $G$. The eccentricity, $\mathrm{ec}_{G}(v)$, of a vertex $v \in V$ is the maximum distance between $v$ and any other vertex in $G$. The maximum distance among all pairs of vertices [2], also known as the value of the maximum eccentricity of the vertices of $G$, is called the diameter of $G$ denoted by $\operatorname{diam}(G)$. The degree, $\operatorname{deg}(v)$, of a vertex $v$ of $G$ is the number of edges incident with $v$. The minimum degree, $\delta(G)=\delta$, of $G$ is the minimum of the degrees of vertices in $G$. The open neighborhood, $N(v)$, of a vertex $v$ is simply the set containing all the vertices adjacent to $v$. The closed neighborhood, $N[v]$, of a vertex $v$ is simply the set containing the vertex $v$ itself and all the vertices adjacent to $v$. We denote by $E\left(V_{1}, V_{2}\right)$ the set $\left\{e=x y \mid x \in V_{1}, y \in V_{2}\right\}$ of edges with one end in $V_{1}$ and the other end in $V_{2}$. The edge connectivity, $\lambda(G)=\lambda$, of $G$ is the minimum number of edges whose deletion from $G$ results in a disconnected or trivial graph. A complete graph, $K_{n}$, is a graph in which every vertex is adjacent to every other vertex. The most likely antonym for a complete graph is a null graph, $\mathcal{N}_{n}$, which is a graph containing only vertices and no edges. A bipartite graph is a graph whose vertices can be divided into two disjoint and independent sets $U$ and $V$ such that every edge in $G$ connects a vertex in $U$ to a vertex in $V$; furthermore, no two vertices in the same set are adjacent to each other. A graph is triangle-free if it does not contain $C_{3}$ as a subgraph and $C_{4}$-free if it does not contain $C_{4}$ as a subgraph. It is important to observe from the above definitions that every bipartite graph is triangle free, but there are some trianglefree graphs which are not bipartite, for example, a cycle graph with five vertices $\left(C_{5}\right)$. For notions not defined here, we refer the reader to [3].

Our motivation for this paper comes from the results published by Erdős et al. in [4] and Mukwembi in [5].

Graphs with forbidden subgraphs are a big part of graph theory literature such as in [4-8]. All graphs in this paper forbid cycle $C_{3}$ as a subgraph. In this paper, we are concerned, in part, with upper bounds on the diameter of bipartite and triangle-free graphs with prescribed edge connectivity in terms of order. The diameter is the most common of the classical distance parameters in graph theory, and much of the research on distances is in fact on the diameter [9]. Several upper bounds on diameter in terms of order and other graph parameters are known, and we list a 
few relevant results below. A well-known and easy to recall result is $1 \leq \operatorname{diam}(G) \leq n-1$. Needless to say, this bound is not only tight for ordinary graphs but also for the field of vision of this paper whenever $\lambda=1$ with the extremal graph being any path, $P_{n}$, on $n$ vertices. Erdös et al. [4] found out that if $G$ is a connected graph of order $n$ and minimum degree $\delta \geq 2$, then

$$
\operatorname{diam}(G) \leq \frac{3 n}{\delta+1}-1,
$$

and they also constructed graphs that, apart from the additive constant, attain the bound. They went further in the same paper and investigated triangle-free and $C_{4}$-free graphs proving that if $G$ is a connected triangle-free graph of order $n$ and minimum degree $\delta \geq 2$, then

$$
\operatorname{diam}(G) \leq 4\left\lceil\frac{n-\delta-1}{2 \delta}\right\rceil .
$$

This can be rewritten as

$$
\operatorname{diam}(G) \leq \frac{2 n}{\delta}+1
$$

In the same paper, they asserted that if $G$ is a connected $C_{4}$-free graph of order $n$ and minimum degree $\delta \geq 2$, then

$$
\operatorname{diam}(G) \leq \frac{5 n}{\delta^{2}-2[\delta / 2]+1} .
$$

Mukwembi [5] investigated $\lambda$-edge-connected graphs and discovered, amongst other bounds, that if $G$ is a $\lambda$-edgeconnected graph of order $n$, then

$$
\operatorname{diam}(G) \leq \frac{3 n}{\lambda+1}-1
$$

Let $x, y \in V$ be fixed vertices in $G$ such that $d(x, y)=$ $\operatorname{diam}(G)=d$ and let $N_{i}=\left\{v \in V \mid d_{G}(x, v)=i\right\}$ for any $0 \leq i \leq d$ and $k_{i}=\left|N_{i}\right|$. The following observation by Mukwembi [5] is essential to this paper.

1.1. Observation. Let $G$ be a $\lambda$-edge-connected graph, $V_{1}, V_{2} \subset V \quad$ with $\quad V_{1} \cap V_{2}=\varnothing$. Clearly, $\left|E\left(V_{1}, V_{2}\right)\right| \leq\left|V_{1}\right|\left|V_{2}\right|$. If $E\left(V_{1}, V_{2}\right)$ is a disconnecting set of $G$, then $\left|E\left(V_{1}, V_{2}\right)\right| \geq \lambda(G)$ so that $\left|V_{1}\right|\left|V_{2}\right| \geq \lambda(G)$. Let $v \in V$. Note also that for $i=0,1, \ldots, \mathrm{ec}_{G}(v)-1$, it is clear that $E\left(N_{i}, N_{i+1}\right)$ is a disconnecting set of $G$.

The following fact follows from the above observation.

Fact 1. $k_{i} k_{i+1} \geq \lambda$ for all $i=1,2, \ldots, \mathrm{ec}_{G}(v)-1$.

The following useful facts follow from the well-known AM-GM inequality $a b \leq((a+b) / 2)^{2}$. That is to say, the geometric mean of two (positive) real numbers never exceeds their arithmetic mean.

Fact 2. For positive integers $a$ and $b$, if $a b \geq 2$, then $a+b \geq 3$.

Fact 3. For positive integers $a$ and $b$, if $a b \geq 5$, then $a+b \geq 5$.

It is the purpose of this paper to bound the diameter of any triangle-free graph with respect to order and edge connectivity. We have dealt with the case wherein $\lambda=1$ and we now proceed to higher values of the same.

Theorem 1. Let $G$ be a triangle-free graph of order $n$ and diameter $d \geq 1$. If $\lambda=2$, then

$$
\operatorname{diam}(G) \leq \frac{2 n}{3}-1
$$

Further, this inequality is best possible.

Proof. Note that since $G$ is 2-edge-connected by the condition of the lemma, we have $k_{i} k_{i+1} \geq 2$ from Fact 1 . From this and Fact 2 , we have $k_{i}+k_{i+1} \geq 3$. Thus, we have two cases.

Case 1. $d$ is even.

$$
\begin{aligned}
n & =\sum_{i=0}^{d} k_{i}=\sum_{i=0}^{(d-2) / 2}\left(k_{2 i}+k_{2 i+1}\right)+k_{d} \\
& \geq \sum_{i=0}^{(d-2) / 2}(3)+1=\frac{3 d}{2}+1,
\end{aligned}
$$

and making $d$ subject of the formula, we obtain $d \leq(2 n / 3)-(2 / 3)$.

Case 2. $d$ is odd.

$$
\begin{aligned}
n & =\sum_{i=0}^{d} k_{i}=\sum_{i=0}^{(d-1) / 2}\left(k_{2 i}+k_{2 i+1}\right) \\
& \geq \sum_{i=0}^{(d-1) / 2}(3)=\frac{3(d+1)}{2},
\end{aligned}
$$

and making $d$ subject of the formula, we obtain $d \leq(2 n / 3)-1$, thereby completing our proof. To show that this bound is asymptotically sharp, consider the following graph: for positive integers $\pi_{0}, \pi_{1}, \ldots, \pi_{d}$, $P_{d}\left(\pi_{0}, \pi_{1}, \ldots, \pi_{d}\right)$ is the graph obtained from a path, $P_{d+1}$, with $d+1$ vertices, by replacing every vertex $v_{i}$ by the null graph $\mathcal{N}_{\pi_{i}}$, where

$$
\pi_{i}= \begin{cases}1, & \text { if } i=0 \text { or } d \\ 2, & \text { if } i=1 \text { or } d-1, \\ 1, & \text { if } i \equiv 0 \bmod (2), \\ 2, & \text { if } i \equiv 1 \bmod (2),\end{cases}
$$

and making every vertex in $\mathcal{N}_{\pi_{q}}$ adjacent to every vertex in $\mathcal{N}_{\pi_{s}}$ whenever $\mathcal{N}_{\pi_{q}}$ and $\mathcal{N}_{\pi_{s}}^{\pi_{q}}$ have replaced adjacent vertices of $P_{d+1}$. Note that $P_{d}\left(\pi_{0}, \pi_{1}, \cdots, \pi_{d}\right)$ is a 2 edge-connected triangle-free graph and that whenever $d$ is even, $\operatorname{diam}\left(P_{d}\left(\pi_{0}, \pi_{1}, \cdots, \pi_{d}\right)\right)=((2 n-2) / 3)$.

The following corollary to Theorem 1 follows from the fact that every bipartite graph is also triangle free and the observation that the corresponding extremal graph for the same theorem is bipartite. 
Corollary 1. Let $G$ be a bipartite graph of order $n$ and diameter $d \geq 1$. If $\lambda=2$, then

$$
\operatorname{diam}(G) \leq \frac{2 n}{3}-1 .
$$

Further, this inequality is best possible.

Although it is not necessarily the scope of this paper, we point out that the above bound also holds and is tight for graphs which are not triangle free, with the same extremal graph being applicable. Mukwembi [5] investigated graphs with edge connectivity $\lambda \in\{3,4\}$ and came up with the following.

Theorem 2. Let $G$ be a $\lambda$-edge-connected graph, $\lambda \in\{3,4\}$, of order $n$; then, $\operatorname{diam}(G) \leq\lfloor(n-1) / 2\rfloor$. Further, this inequality is best possible with the exception of a small constant.

While this bound is for graphs which are unrestricted with respect to subgraphs, it is also tight for both bipartite and triangle-free graphs. To see this, consider the graph $P_{d}\left(\pi_{0}, \pi_{1}, \ldots, \pi_{d}\right)$ with all the same properties as before, except that

$$
\pi_{i}= \begin{cases}1, & \text { if } i=0 \text { or } d, \\ \lambda, & \text { if } i=1 \text { or } d-1 \\ \lambda-1, & \text { if } i=2 \text { or } d-2 \\ 2, & \text { otherwise }\end{cases}
$$

Let $\operatorname{diam}\left(P_{d}\left(\pi_{0}, \pi_{1}, \ldots, \pi_{d}\right)\right)=d$. Observe that $P_{d}\left(\pi_{0}, \pi_{1}, \ldots, \pi_{d}\right)$ is bipartite and triangle free and that if $\lambda=3$, then $d=((n-2) / 2), \quad$ and if $\lambda=4$, then $d=((n-6) / 2)$. The case where $\lambda=5$ is rather atypical and requires added attention; hence, we earmark it for one of the main results in our paper. For $\lambda \geq 6$, we have the following.

Theorem 3. Let $G$ be a triangle-free, $\lambda$-edge-connected graph, $\lambda \geq 6$, of order $n$. Then,

$$
\operatorname{diam}(G) \leq \frac{2 n}{\lambda}+1
$$

Further, this inequality is best possible with the exception of a small constant.

Proof. An application of the Whitney inequality, $\delta \geq \lambda$, to equation (3) yields the desired result. Now, let $p \in \mathbb{N}$, be a number such that it is the least number satisfying the inequality $\lambda \leq p^{2}$. To show that this bound is tight, consider the graph $P_{d}\left(\pi_{0}, \pi_{1}, \ldots, \pi_{d}\right)$ with all the same properties as before, except that

$$
\pi_{i}= \begin{cases}1, & \text { if } i=0 \text { or } d \\ \lambda, & \text { if } i=1 \text { or } d-1, \\ \lambda-1, & \text { if } i=2 \text { or } d-2, \\ p, & \text { if } i \equiv 0 \text { or } 1 \bmod (4), \\ \lambda-p, & \text { if } i \equiv 2 \text { or } 3 \bmod (4) .\end{cases}
$$

Observe $P_{d}\left(\pi_{0}, \pi_{1}, \cdots, \pi_{d}\right)$ is a $\lambda$-edge-connected triangle-free graph and that whenever $d \equiv 1 \bmod (4)$, then $\operatorname{diam}\left(P_{d}\left(\pi_{0}, \pi_{1}, \cdots, \pi_{d}\right)\right)=(2 n / \lambda)-3$.
The following corollary to Theorem 3 follows from the fact that every bipartite graph is also triangle free and the observation that the corresponding extremal graph for the same theorem is bipartite.

Corollary 2. Let $G$ be a $\lambda$-edge-connected, bipartite graph, $\lambda \geq 6$, of order $n$. Then,

$$
\operatorname{diam}(G) \leq \frac{2 n}{\lambda}+1 .
$$

Further, this inequality is best possible with the exception of a small constant.

This paper also aims to bound the diameter of any bipartite or triangle-free graph with respect to size and edge connectivity. It is clear that the bound $\operatorname{diam}(G) \leq(m / \lambda)$ holds for whatever value of $\lambda$ we choose. This bound, however, is only tight when $\lambda \in\{1,2,4\}$, with the extremal graphs for these values of $\lambda$ being the same as those offered up for similar values of $\lambda$ when we were discussing bounds on diameter with respect to order and edge connectivity. For $\lambda \geq 6$, the following theorem, which at first glance seems counter intuitive, holds true.

Theorem 4. Let $G$ be a $\lambda$-edge-connected, $\lambda \geq 6$, triangle-free graph of size $m$; then, $\operatorname{diam}(G) \leq\left(4 m / \lambda^{2}\right)+1$. Further, this inequality is best possible with the exception of a small constant.

Proof. An application of the Whitney inequality, $\delta \geq \lambda$, and the handshaking lemma, $\sum_{x \in V} \operatorname{deg}(x)=2 m$, yields the inequality $n \lambda \leq 2 m$, and applying this to Theorem 3 , we obtain the desired result. The extremal graph for this bound is the same as the one for Theorem 3 and has diameter $\operatorname{diam}(G)=$ $\left(4 m / \lambda^{2}\right)-2$ whenever $\operatorname{diam}(G) \equiv 2 \bmod (4)$.

The following corollary to Theorem 4 follows from the fact that every bipartite graph is also triangle free and the observation that the corresponding extremal graph for the same theorem is bipartite.

Corollary 3. Let $G$ be a $\lambda$-edge-connected bipartite graph of size $m$; then, $\operatorname{diam}(G) \leq\left(4 m / \lambda^{2}\right)+1$. Further, this inequality is best possible with the exception of a small constant.

The cases where $\lambda=3$ and $\lambda=5$ need more care and as such have been allotted space among our main results.

\section{Results}

2.1. 3-Edge-Connected Graphs. Let $G$ be a 3-edge-connected triangle-free graph of size $m$ and diameter $d>1$.

For an example of a 3-edge-connected graph, consider the following graph: for positive integers $\pi_{0}, \pi_{1}, \ldots, \pi_{d}$, $P_{d}\left(\pi_{0}, \pi_{1}, \ldots, \pi_{d}\right)$ is the graph obtained from a path, $P_{d+1}$, with $d+1$ vertices, by replacing every vertex $v_{i}$ by the null graph $\mathcal{N}_{\pi}$, where

$$
\pi_{i}= \begin{cases}1, & \text { if } i=0 \text { or } d \\ 3, & \text { otherwise }\end{cases}
$$


and making every vertex in $\mathcal{N}_{\pi_{q}}$ adjacent to every vertex in $\mathcal{N}_{\pi_{s}}$ whenever $\mathcal{N}_{\pi_{q}}$ and $\mathcal{N}_{\pi_{s}}$ have replaced adjacent vertices of $P_{d+1}$.

Let $x, y \in V$ be fixed vertices in $G$ such that $d(x, y)=\operatorname{diam}(G)=d$. For any $0 \leq i \leq d$, let $N_{i}=\left\{v \in V \mid d_{G}(x, v)=i\right\}$ and $k_{i}=\left|N_{i}\right|$. For any $0 \leq j, l \leq d$, where $j<l$, let $M_{[j, l]}=\left\{e \in E \mid e \in E\left(\left(\cup_{i=j}^{l} N_{i}\right),\left(\cup_{i=j}^{l} N_{i}\right)\right)\right\}$ and $m_{[j, l]}=\left|M_{[j, l]}\right|$. Also, let $M_{i}$ be the set of edges between vertices in the same distance layer, that is to say, $M_{i}=E\left(N_{i}, N_{i}\right)$, and let the cardinality of the same set be given by $m_{i}=\left|M_{i}\right|$. Further, $M_{(j, l)}=M_{[j, l]}-\left(M_{j} \cup M_{l}\right)$ and $m_{(j, l)}=\left|M_{(j, l)}\right|$.

Lemma 1. For each $i \in\{1,2, \ldots, d-1\}$, if $k_{i} \geq 2$, then $m_{(i-1, i+1)} \geq 7$.

Proof. Since $G$ is a 3-edge-connected graph, we have that $m_{(i-1, i)}, m_{(i, i+1)} \geq 3$ so that if $M_{i}$ is nonempty, we are immediately satisfied since $m_{(i-1, i+1)}=m_{(i-1, i)}+m_{(i, i+1)}+$ $m_{i} \geq 3+3+1=7$. Hence, suppose that $M_{i}$ is empty. Suppose also, to the contrary, that $m_{(i-1, i+1)}<7$. Since $G$ is 3-edge-connected, we see that $m_{(i-1, i+1)} \geq m_{(i-1, i)}+$ $m_{(i, i+1)} \geq 3+3=6$. Thus, by our supposition, $m_{(i-1, i+1)}=6$ and $m_{(i-1, i)}=m_{(i, i+1)}=3$. Now, choose any two vertices in $N_{i}$, say $a$ and $b$. Note that $\operatorname{deg}(a), \operatorname{deg}(b) \geq 3$ since $G$ is 3 edge-connected. Further $a$ and $b$ share no edges since $M_{i}$ is empty; thus, of the six edges in $M_{(i-1, i+1)}$, we have that three are incident with $a$ and the other three are incident with $b$. Also, since there necessarily exists a path from $N_{i-1}$ to $N_{i+1}$ and $m_{(i-1, i)}=m_{(i, i+1)}=3$, we are guaranteed that $a$ and $b$ are each incident with at least an edge in each of the sets $M_{(i-1, i)}$ and $M_{(i, i+1)}$. And since for each of $a$ and $b$ there are three edges and two sets, it is necessary that one of the edges incident with $a$ (or $b$ ) be in a set different from the other two edges incident with $a$ (or $b$ ). For $a$, label this edge $e_{a}$, and for $b$, label this edge $e_{b}$. Observe that $G-\left\{e_{a}, e_{b}\right\}$ is disconnected contradicting our supposition that $G$ is 3-edge-connected. This immediately settles our lemma.

Lemma 2. For each $i \in\{1,2, \cdots, d-3\}$, if $m_{(i-1, i+1)}=6$, then $m_{(i+1, i+3)} \geq 9$.

Proof. By the condition of the current lemma and by Lemma 1 , we have $k_{i}=1$. From this and again using the condition of the current lemma combined with the fact that $G$ is 3-edgeconnected, we see that $k_{i-1}=k_{i+1}=3$ and that $m_{(i-1, i)}=m_{(i, i+1)}=3$. Note also that $M_{i+1}$ is empty since $G$ is triangle free and all the vertices in $N_{i+1}$ are adjacent to the single vertex in $N_{i}$. Hence, the vertices in $N_{i+1}$ share no edges and consequently $m_{(i, i+1)}+m_{(i+1, i+2)} \geq \delta\left(k_{i+1}\right)=9$ which yields $m_{(i+1, i+2)} \geq 9-m_{(i, i+1)}=6$. Note that $m_{(i+2, i+3)} \geq 3$ since $G$ is 3 -edge-connected, and hence $m_{(i+1, i+3)} \geq m_{(i+1, i+2)}+m_{(i+2, i+3)} \geq 6+3=9$, thereby proving our lemma.
Lemma 3. For each $i \in\{3,4, \ldots, d-1\}$, if $m_{(i-1, i+1)}=6$, then $m_{(i-3, i-1)} \geq 9$.

Proof. We can get the desired conclusion by arguing as in the proof of Lemma 2.

Lemma 4. For each $i \in\{2,4, \ldots, d-2\}, m_{(i-2, i+2)} \geq 14$.

Proof. Note that since $\lambda=3$, we have that $m_{(i-2, i)} \geq m_{(i-2, i-1)}+m_{(i-1, i)} \geq 3+3=6 . \quad$ Also, $m_{(i, i+2)} \geq m_{(i, i+1)}+m_{(i+1, i+2)} \geq 3+3=6$. Hence, we obtain the following:

If $m_{(i-2, i)}, m_{(i, i+2)} \geq 7$, we have $m_{(i-2, i+2)} \geq m_{(i-2, i)}+$ $m_{(i, i+2)} \geq 7+7=14$ and we are done.

If $m_{(i-2, i)}=6$, then we have, by Lemma 2, $m_{(i-2, i+2)} \geq m_{(i-2, i)}+m_{(i, i+2)} \geq 6+9=15$ and we are done.

If $m_{(i, i+2)}=6$, then we have, by Lemma 3 , $m_{(i-2, i+2)} \geq m_{(i-2, i)}+m_{(i, i+2)} \geq 9+6=15$, thereby settling our lemma.

Throughout the rest of this result we define $c \in\{0,1,2,3\}$ as a number such that $d \equiv c \bmod 4$ where $d=\operatorname{diam}(G)$.

The following theorem provides a tight upper bound on the diameter of a 3-edge-connected triangle-free graph of prescribed size.

Theorem 5. Let $G$ be a 3-edge-connected triangle-free graph of size $m$. Then,

$$
\operatorname{diam}(G) \leq \frac{2 m+3}{7}
$$

This inequality is, apart from an additive constant, best possible.

Proof. Note that $m=m_{[0, d]} \geq \sum_{i=0}^{(d-c-4) / 4} m_{(4 i, 4 i+4)}+$ $\left(m_{(d-c, d-c+1)}+m_{(d-c+1, d-c+2)}+m_{(d-c+2, d-c+3)}\right)$ where $m_{(i, i+1)}=$ 0 whenever $i \geq d$. Hence, there are four cases to consider.

Case 1. $d \equiv 0 \bmod 4$. If this is so, then $c=0$, and we have, by Lemma 4,

$$
\begin{aligned}
m & =m_{[0, d]} \geq \sum_{i=0}^{(d-4) / 4} m_{(4 i, 4 i+4)} \\
& \geq \sum_{i=0}^{(d-4) / 4}(14)=\frac{14 d}{4},
\end{aligned}
$$

and making $d$ subject of the formula, we obtain $d \leq(2 m / 7)$.

Case 2. $d \equiv 1 \bmod 4$. If this is so, then $c=1$, and we have the following by Lemma 4 and the fact that $m_{(i, i+1)} \geq \lambda=3$ for all $i<d$ : 


$$
\begin{aligned}
m & =m_{[0, d]} \geq \sum_{i=0}^{(d-5) / 4} m_{(4 i, 4 i+4)}+m_{(d-1, d)} \\
& \geq \sum_{i=0}^{(d-5) / 4}(14)+3=\frac{14(d-1)}{4}+3
\end{aligned}
$$

and making $d$ subject of the formula, we obtain $d \leq((2 m+1) / 7)$.

Case 3. $d \equiv 2 \bmod 4$. If this is so, then $c=2$, and we have the following by Lemma 4 and the fact that $m_{(i, i+1)} \geq \lambda=3$ for all $i<d$ :

$$
\begin{aligned}
m & =m_{[0, d]} \geq \sum_{i=0}^{(d-6) / 4} m_{(4 i, 4 i+4)}+m_{(d-2, d-1)}+m_{(d-1, d)} \\
& \geq \sum_{i=0}^{(d-6) / 4}(14)+3+3=\frac{14(d-2)}{4}+6
\end{aligned}
$$

and making $d$ subject of the formula, we obtain $d \leq((2 m+2) / 7)$.

Case 4. $d \equiv 3 \bmod 4$. If this is so, then $c=3$, and we have the following by Lemma 4 and the fact that $m_{(i, i+1)} \geq \lambda=3$ for all $i<d$ :

$$
\begin{aligned}
m= & m_{[0, d]} \geq \sum_{i=0}^{(d-7) / 4} m_{(4 i, 4 i+4)}+m_{(d-3, d-2)}+m_{(d-2, d-1)} \\
& +m_{(d-1, d)} \\
\geq & \sum_{i=0}^{(d-7) / 4}(14)+3+3+3=\frac{14(d-3)}{4}+9,
\end{aligned}
$$

and making $d$ subject of the formula, we obtain $d \leq((2 m+3) / 7)$.

Hence, considering all four cases, we obtain $d \leq((2 m+$ 3)/7) as desired. To show that this bound is tight, consider the graph $P_{d}\left(\pi_{0}, \pi_{1}, \ldots, \pi_{d}\right)$ with all the same properties as before, except that

$$
\begin{gathered}
\pi_{i}= \begin{cases}1, & \text { if } i=0 \text { or } d, \\
3, & \text { if } i=1 \text { or } d-1, \\
2, & \text { otherwise, }\end{cases} \\
m_{(i, i+1)}= \begin{cases}3, & \text { if } i=0 \text { or } d-1, \\
6, & \text { if } i=1 \text { or } d-2, \\
3, & \text { if } i \text { is even }, \\
4, & \text { if } i \text { is odd } .\end{cases}
\end{gathered}
$$

Observe that whenever $d$ is even, $\operatorname{diam}\left(P_{d}\left(\pi_{0}, \pi_{1}, \ldots, \pi_{d}\right)\right)=((2 m-8) / 7)$.
The following corollary to Theorem 5 follows from the fact that every bipartite graph is also triangle free and the observation that the corresponding extremal graph for the same theorem is bipartite.

Corollary 4. Let $G$ be a 3-edge-connected bipartite graph of size $m$. Then,

$$
\operatorname{diam}(G) \leq \frac{2 m+3}{7}
$$

This inequality is, apart from an additive constant, best possible.

The following definitions and lemmas will be used in the study of 5-edge-connected graphs.

2.2. 5-Edge-Connected Graphs. Let G be a 5-edge-connected triangle-free graph of order $n$ and diameter $d>1$.

For an example of a 5-edge-connected graph, consider the following graph: for positive integers $\pi_{0}, \pi_{1}, \cdots, \pi_{d}$, $P_{d}\left(\pi_{0}, \pi_{1}, \cdots, \pi_{d}\right)$ is the graph obtained from a path, $P_{d+1}$, with $d+1$ vertices, by replacing every vertex $v_{i}$ by the null graph $\mathcal{N}_{\pi_{i}}$, where

$$
\pi_{i}= \begin{cases}1, & \text { if } i=0 \text { or } d \\ 5, & \text { otherwise }\end{cases}
$$

and making every vertex in $\mathcal{N}_{\pi_{q}}$ adjacent to every vertex in $\mathcal{N}_{\pi_{s}}$ whenever $\mathcal{N}_{\pi_{q}}$ and $\mathcal{N}_{\pi_{s}}$ have replaced adjacent vertices of $P_{d+1}$.

Let $x, y \in V$ be fixed vertices in $G$ such that $d(x, y)=$ $\operatorname{diam}(G)=d$ and let $N_{i}=\left\{v \in V \mid d_{G}(x, v)=i\right\}$ for any $0 \leq i \leq d$ and $k_{i}=\left|N_{i}\right|$.

Lemma 5. For each $i \in\{0,1, \cdots, d-1\}, k_{i}+k_{i+1} \geq 5$.

Proof. Note that since $G$ is 5-edge-connected, we have $k_{i} k_{i+1} \geq 5$ by Fact 1 . Because of this and as a consequence of Fact 3 , we obtain $k_{i}+k_{i+1} \geq 5$.

The following two lemmas follow immediately from Lemma 5.

Lemma 6. For $i \in\{1,2, \cdots, d-1\}$, if $k_{i}=1$, then $k_{i-1}+k_{i}+k_{i+1} \geq 9$.

Lemma 7. For $i \in\{1,2, \cdots, d-1\}$, if $k_{i}=2$, then $k_{i-1}+k_{i}+k_{i+1} \geq 8$.

Lemma 8. For $i \in\{1,2, \cdots, d-1\}$, if $k_{i} \geq 3$, then $k_{i-1}+k_{i}+k_{i+1} \geq 8$.

Proof. There are two cases to consider.

Case 1. There exists an edge, $a b \in E$, such that $a, b \in N_{i}$. Since $G$ is triangle free, the effect of this is that $a$ and $b$ share no neighbors, that is to say, $N(a) \cap N(b)=\varnothing$. Note that $N(a) \cup N(b) \subseteq N_{i-1} \cup N_{i} \cup N_{i+1}$ and $|N(a)|,|N(b)| \geq \delta \geq \lambda=5$. Hence, $k_{i-1}+k_{i}+k_{i+1} \geq$ $|N(a) \cup N(b)|=|N(a)|+|N(b)| \geq 5+5=10$. 
Case 2. There are no adjacent vertices in $N_{i}$.

Let $a \in N_{i}$. Since there are no adjacent vertices in $N_{i}$, we see that $N(a) \cap N_{i}=\varnothing$. Also, $N(a) \subseteq N_{i-1} \cup N_{i+1}$ and $|N(a)| \geq \delta \geq \lambda=5$. Hence, $\quad k_{i-1}+k_{i}+$ $k_{i+1} \geq\left|N_{i} \cup N(a)\right|=\left|N_{i}\right|+|N(a)| \geq 3+5=8$.

Lemma 9. For each $i \in\{1,2, \cdots, d-1\}, k_{i-1}+k_{i}+k_{i+1} \geq 8$.

Proof. There are three cases to consider.

Case 1. $k_{i}=1$.

If this is so, then we are done by Lemma 6 .

Case 2. $k_{i}=2$.

If this is so, then we are done by Lemma 7.

Case 3. $k_{i} \geq 3$.

If this is so, then we are done by Lemma 8 .

Throughout the rest of this paper we define $c \in\{0,1,2\}$ as a number such that $d-c \equiv 2 \bmod 3$ where $d=\operatorname{diam}(G)$.

The following theorem provides a tight upper bound on the diameter of a 5-edge-connected triangle-free graph of prescribed order.

Theorem 6. Let $G$ be a 5-edge-connected triangle-free graph of order $n$. Then,

$$
\operatorname{diam}(G) \leq \frac{3 n-3}{8}
$$

This inequality is, apart from an additive constant, best possible.

Proof. Note that $n=\sum_{i=0}^{d} k_{i}=\sum_{i=0}^{(d-c-2) / 3}\left(k_{3 i}+k_{3 i+1}+k_{3 i+2}\right)+$ $\left(k_{d-c+1}+k_{d-c+2}\right)$ where $k_{i}=0$ whenever $i>d$. Hence, there are three cases to consider.

Case 1. $d \equiv 0 \bmod 3$. If this is so, then $c=1$, and we have the following by Lemma 9 and the fact that $k_{d} \geq 1$ since $N_{d}$ is nonempty:

$$
\begin{aligned}
n & =\sum_{i=0}^{d} k_{i}=\sum_{i=0}^{(d-1-2) / 3}\left(k_{3 i}+k_{3 i+1}+k_{3 i+2}\right)+k_{d} \\
& \geq \sum_{i=0}^{(d-3) / 3}\left(k_{3 i}+k_{3 i+1}+k_{3 i+2}\right)+k_{d} \geq \sum_{i=0}^{(d-3) / 3}(8)+1 \\
& =\left(\frac{d-3}{3}+1\right)(8)+1=\frac{8 d}{3}+1,
\end{aligned}
$$

and making $d$ subject of the formula, we obtain $d \leq((3 n-3) / 8)$.

Case 2. $d \equiv 1 \bmod 3$. If this is so, then $c=2$, and we have the following by Lemma 9 and Lemma 5:

$$
\begin{aligned}
n & =\sum_{i=0}^{d} k_{i}=\sum_{i=0}^{(d-2-2) / 3}\left(k_{3 i}+k_{3 i+1}+k_{3 i+2}\right)+\left(k_{d-1}+k_{d}\right) \\
& \geq \sum_{i=0}^{(d-4) / 3}\left(k_{3 i}+k_{3 i+1}+k_{3 i+2}\right)+\left(k_{d-1}+k_{d}\right) \\
& \geq \sum_{i=0}^{(d-4) / 3}(8)+5=\left(\frac{d-4}{3}+1\right)(8)+5=\frac{8 d-8}{3}+5,
\end{aligned}
$$

and making $d$ subject of the formula, we obtain $d \leq((3 n-7) / 8)$.

Case 3. $d \equiv 2 \bmod 3$. If this is so, then $c=0$, and we have the following by Lemma 9:

$$
\begin{aligned}
n & =\sum_{i=0}^{d} k_{i}=\sum_{i=0}^{(d-0-2) / 3}\left(k_{3 i}+k_{3 i+1}+k_{3 i+2}\right) \\
& \geq \sum_{i=0}^{(d-2) / 3}\left(k_{3 i}+k_{3 i+1}+k_{3 i+2}\right) \geq \sum_{i=0}^{(d-2) / 3}(8) \\
& =\left(\frac{d-2}{3}+1\right)(8)=\frac{8 d+8}{3},
\end{aligned}
$$

and making $d$ subject of the formula, we obtain $d \leq(3 n / 8)-1$.

Hence, considering all three cases, we obtain $d \leq((3 n-$ 3)/8) as desired. To show that this bound is tight, consider the graph $P_{d}\left(\pi_{0}, \pi_{1}, \cdots, \pi_{d}\right)$ with all the same properties as before, except that

$$
\pi_{i}= \begin{cases}1, & \text { if } i=0 \text { or } d, \\ 5, & \text { if } i=1 \text { or } d-1, \\ 4, & \text { if } i=2 \text { or } d-2, \\ 2, & \text { if } i \equiv 0 \bmod 3, \\ 3, & \text { if } i \equiv 1 \text { or } 2 \bmod 3 .\end{cases}
$$

Observe that whenever $d \equiv 2 \bmod 3$, $\operatorname{diam}\left(P_{d}\left(\pi_{0}, \pi_{1}, \cdots, \pi_{d}\right)\right)=((3 n-20) / 8)$.

The following corollary to Theorem 6 follows from the fact that every bipartite graph is also triangle free and the observation that the corresponding extremal graph for the same theorem is bipartite.

Corollary 5. Let $G$ be a 5-edge-connected bipartite graph of order $n$. Then,

$$
\operatorname{diam}(G) \leq \frac{3 n-3}{8}
$$

This inequality is, apart from an additive constant, best possible.

Our final result is a tight upper bound on the diameter of a 5-edge-connected bipartite graph with respect to its size. To obtain the said bound, we need the following definitions 
and lemmas. Let $G$ be a 5-edge-connected bipartite graph of size $m$ and diameter $d>1$. Let $x, y \in V$ be fixed vertices in $G$ such that $d(x, y)=\operatorname{diam}(G)=d$ and let $N_{i}=\left\{v \in V \mid d_{G}(x, v)=i\right\}$ for any $0 \leq i \leq d$ and $k_{i}=\left|N_{i}\right|$. For $j<l$, let $M_{[j, l]}=\left\{e \in E \mid e \in E\left(\left(\cup_{i=j}^{l} N_{i}\right),\left(\cup_{i=j}^{l} N_{i}\right)\right)\right\}$ for any $0 \leq j \leq d-1$ and $m_{[j, l]}=\left|M_{[j, l]}\right|$. Also, let $M_{(j, l)}=M_{[j, l]}-$ $\left(E\left(N_{j}, N_{j}\right) \cup E\left(N_{l}, N_{l}\right)\right)$ and $m_{(j, l)}=\left|M_{(j, l)}\right| . M_{i}$ is simply the set of edges between vertices in the same distance layer, that is to say, $M_{i}=E\left(N_{i}, N_{i}\right)$, and the cardinality of the same set is given by $m_{i}=\left|M_{i}\right|$.

Lemma 10. For each $i \in\{0,1, \cdots, d\}, M_{i}$ is empty.

Proof. The desired conclusion follows from the assumption that $G$ is bipartite.

Lemma 11. For each $i \in\{1, \cdots, d-1\}$, if $k_{i+1}+k_{i+2} \geq 6$, then $m_{(i, i+3)} \geq 21$.

Proof. Note that, by Lemma 10, $M_{i}=\varnothing$. Since $k_{i+1}+k_{i+2} \geq 6$, choose any six vertices from $N_{i+1} \cup N_{i+2}$. Label the set of vertices we have chosen from $N_{i+1}$ as $N_{i+1}^{\prime}$ and the set of vertices we have chosen from $N_{i+2}$ as $N_{i+2}^{\prime}$. Identify the edges incident to vertices in $N_{i+1}^{\prime}$ and $N_{i+2}^{\prime}$ as $E^{I}\left(N_{i+1}^{\prime}\right)$ and $E^{I}\left(N_{i+2}^{\prime}\right)$, respectively. Note that $m_{(i, i+3)} \geq\left|E^{I}\left(N_{i+1}^{\prime}\right) \cup E^{I}\left(N_{i+2}^{\prime}\right)\right|=$

$\left|E^{I}\left(N_{i+1}^{\prime}\right)\right|+\left|E^{I}\left(N_{i+2}^{\prime}\right)\right|-\left|E^{I}\left(N_{i+1}^{\prime}\right) \cap E^{I}\left(N_{i+2}^{\prime}\right)\right|$. Observe that since $\left|N_{i+1}^{\prime}\right|+\left|N_{i+2}^{\prime}\right|=6$ and since $\delta \geq \lambda \geq 5$, we have, by Lemma 10, that $\left|E^{I}\left(N_{i+1}^{\prime}\right)\right|+\left|E^{I}\left(N_{i+2}^{\prime}\right)\right| \geq \sum_{x \in N_{i+1}^{\prime}} \cup N_{i+2}^{\prime} \operatorname{deg}(x) \geq 6 \delta=30$.

Since $N_{i+1}^{\prime} \cap N_{i+2}^{\prime}=\varnothing$ and using the AM-GM inequality, we observe that $\left|E^{I}\left(N_{i+1}^{\prime}\right) \cap E^{I}\left(N_{i+2}^{\prime}\right)\right|=\left|E\left(N_{i+1}^{\prime}, N_{i+2}^{\prime}\right)\right| \leq$ $\left|N_{i+1}^{\prime}\right|\left|N_{i+2}^{\prime}\right| \leq\left(\left(\left|N_{i+1}^{\prime}\right|+\left|N_{i+2}^{\prime}\right|\right) / 2\right)^{2}=9 . \quad$ Hence, $m_{(i, i+3)} \geq\left|E^{I}\left(N_{i+1}^{\prime}\right) \cup E^{I}\left(N_{i+2}^{\prime}\right)\right|=\left|E^{I}\left(N_{i+1}^{\prime}\right)\right|+\left|E^{I}\left(N_{i+2}^{\prime}\right)\right|-$ $\left|E^{I}\left(N_{i+1}^{\prime}\right) \cap E^{I}\left(N_{i+2}^{\prime}\right)\right| \geq 30-9=21$, and we are done.

The following fact stems from Lemma 5 and the observation that every bipartite graph is also triangle free.

Fact 4. For each $i \in\{0,1, \cdots, d-1\}, k_{i}+k_{i+1} \geq 5$.

For $i \in\{1,2, \cdots, d-3\}$, let $S$ be an induced subgraph of $G$ such that $V(S)=\cup_{j=i}^{i+3} N_{j}$. We then have the following useful definition.

Definition 1. $S$ is a scant subgraph of $G$ (or simply, $S$ is scant) if $m_{(i, i+3)}<21$.

Lemma 12. If $S$, where $V(S)=\cup_{j=i}^{i+3} N_{j}$, is scant, then $\left(k_{i+1}, k_{i+2}\right)=(2,3)$ or $\left(k_{i+1}, k_{i+2}\right)=(3,2)$.

Proof. By Fact 4, Lemma 11, and by the condition of the current lemma, we have that $k_{i+1}+k_{i+2}=5$. Hence, $\left(k_{i+1}, k_{i+2}\right) \in\{(1,4),(2,3),(3,2),(4,1)\}$, and it is sufficient to prove the current lemma if we can show that $k_{i+1}, k_{i+2} \neq 1$. Suppose to the contrary that $k_{i+1}=1$. If this is so, then $k_{i+2}=4$, and since $N_{i+1} \cap N_{i+2}=\varnothing$, we obtain $E\left(N_{i+1}, N_{i+2}\right) \leq k_{i+1} k_{i+2}=4<5=\lambda$, contradicting the condition that $G$ is 5-edge-connected. The same argument is to be used for the case where $k_{i+2}=1$, hence settling our lemma.
As a consequence of Lemma 12, we further add the following definitions.

Definition 2. Let $S$, where $V(S)=\cup_{j=i}^{i+3} N_{j}$, be a scant subgraph of $G$. If $\left(k_{i+1}, k_{i+2}\right)=(2,3)$, we say $S$ is scant-left, and if $\left(k_{i+1}, k_{i+2}\right)=(3,2)$, we say $S$ is scant-right.

Lemma 13. If $S$, where $V(S)=\cup_{j=i}^{i+3} N_{j}$, is scant, then $m_{(i, i+3)}=20$.

Proof. By the condition of the current lemma and by Lemma 12 , we have that $\left(k_{i+1}, k_{i+2}\right)=(2,3)$ or $\left(k_{i+1}, k_{i+2}\right)=(3,2)$. Hence, there are two cases to consider.

Case 1. $\left(k_{i+1}, k_{i+2}\right)=(2,3)$.

Note that $m_{(i, i+3)} \geq m_{(i, i+1)}+m_{(i+1, i+3)}$. Note also that since, by Lemma $10, M_{i+2}$ is empty and since $\delta \geq \lambda \geq 5$, we have that $m_{(i+1, i+3)}=\sum_{x \in N_{i+2}} \operatorname{deg}(x) \geq \delta k_{i+2}=15$. Further, observe that $M_{(i, i+1)}$ is a disconnecting set of $G$, and hence $m_{(i, i+1)} \geq \lambda=5$. Thus, we have $m_{(i, i+3)} \geq m_{(i, i+1)}+m_{(i+1, i+3)} \geq 5+15=20$.

Case 2. $\left(k_{i+1}, k_{i+2}\right)=(3,2)$.

We can get the desired conclusion by arguing as in case 1.

\section{Lemma 14}

(a) If $S$, where $V(S)=\cup_{j=i}^{i+3} N_{j}$, is scant-left, then $m_{(i-3, i)} \geq 25$.

(b) If $S$, where $V(S)=\cup_{j=i}^{i+3} N_{j}$, is scant-right, then $m_{(i+3, i+6)} \geq 25$.

Proof. We will show that the result holds for (a) as it follows analogously for (b).

(a) $k_{i+2}=3$ by the condition of the lemma, and hence we have that $m_{(i+1, i+3)} \geq \sum_{x \in N_{i+2}} \operatorname{deg}(x) \geq \delta k_{i+2}=15$. Also, $m_{(i, i+3)} \leq 20$, by the condition of the lemma. Hence, $m_{(i, i+1)} \leq m_{(i, i+3)}-m_{(i+1, i+3)} \leq 20-15=5$. Note that by the condition of the lemma and by Fact 4 , we have $k_{i} \geq 3$. If each vertex in $N_{i}$ is adjacent to both vertices in $N_{i+1}$, then $m_{(i, i+1)} \geq k_{i} k_{i+1} \geq 2 k_{i} \geq 6$ which contradicts our deduction that $m_{(i, i+1)}<6$. Hence, there is at least one vertex, say $a$, in $N_{i}$ which is adjacent to at most one vertex in $N_{i+1}$. By Lemma 10 and since $\operatorname{deg}(a) \geq \delta \geq \lambda \geq 5$, we see that $a$ has at least four neighbors in $N_{i-1}$, that is to say, $k_{i-1} \geq 4$. Note that $m_{(i-3, i)} \geq m_{(i-3, i-2)}+m_{(i-2, i)}$. Note also that since, by Lemma $10, M_{i-1}$ is empty and since $\delta \geq \lambda \geq 5$, we have that $m_{(i-2, i)}=\sum_{x \in N_{i-1}} \operatorname{deg}(x) \geq \delta k_{i-1} \geq 20$. Further, observe that $M_{(i-3, i-2)}$ is a disconnecting set of $G$, and hence $m_{(i-3, i-2)} \geq \lambda=5$. Thus, we have $m_{(i-3, i)} \geq m_{(i-3, i-2)}+m_{(i-2, i)} \geq 5+20=25$.

Throughout the remainder of this paper, we assume that $\operatorname{diam}(G)=d \equiv c \bmod 3, c \in\{0,1,2\}$, and we define the set $I=\{0,1, \cdots,((d-c-3) / 3)\}$. For $p \in I$, we define $S_{p}=\cup_{j=3 p}^{3 p+3} N_{i}$. As a result of the previous lemma, we have the following definition. 
Definition 3. For $p \in I$, we say $S_{p}$ is a surplus subgraph of $G$ (or simply, $S_{p}$ is a surplus) if $m_{(3 p, 3 p+3)} \geq 25$.

Lemma 15. $m_{(0, d-c)} \geq 21|I|$.

Proof. We have three cases to consider.

Case 1. For all $p \in I, S_{p}$ is not scant.

If this is so, then $m_{(0, d-c)} \geq \sum_{p \in I} m_{(3 p, 3 p+3)} \geq 21|I|$ and we are done.

In the remaining two cases, we assume that for at least one $p \in I, S_{p}$ is scant. Let $A=\left\{p_{j} \mid j \in\{1,2, \ldots, \alpha\}\right\}$ and $B=\left\{q_{j} \mid j \in\{1,2, \ldots, \beta\}\right\}$ such that $S_{p}$ is a leftscant whenever $p \in A$ and $S_{p}$ is a right-scant whenever $p \in B$.

Case 2. $\alpha \geq \beta$.

If this is so, we have the following. Let $C_{1}=\{p-1 \mid p \in A\}$; then, by Lemma $14, S_{p}$ is a surplus whenever $p \in C_{1} \subset I$. Also, let $D_{1}=I-\left(A \cup B \cup C_{1}\right)$. Notice that $\left|C_{1}\right|=|A|$ and since $A, B$, and $C_{1}$ are mutually exclusive sets, we have $\left|D_{1}\right|=|I|-|A|-|B|-\left|C_{1}\right|=|I|-2|A|-|B|$. Further, since $\alpha \geq \beta$, we have that $3|A|-|B|>0$. Hence, we obtain $m_{(0, d-c)} \geq \sum_{p \in I} m_{(3 p, 3 p+3)}=\sum_{p \in D_{1}} m_{(3 p, 3 p+3)}+$ $\sum_{p \in A} m_{(3 p, 3 p+3)}+\sum_{p \in B} m_{(3 p, 3 p+3)}+\sum_{p \in C_{1}} m_{(3 p, 3 p+3)} \geq$ $20|A|+20|B|+25\left|C_{1}\right|+21\left|D_{1}\right|=20|A|+20|B|+$

$25|A|+21(|I|-2|A|-|B|)=21|I|+3|A|-|B| \geq 21|I|$ and we are done.

Case 3. $\alpha<\beta$.

We can get the desired conclusion by arguing as in case 2 .

Our final theorem below provides a tight upper bound on the diameter of a 5-edge-connected bipartite graph of prescribed size.

Theorem 7. Let $G$ be a 5-edge-connected bipartite graph of size $m$. Then,

$$
d=\operatorname{diam}(G) \leq \frac{m+4}{7} .
$$

This inequality is, apart from an additive constant, best possible.

Proof. There are three cases to consider.

Case 1. $d \equiv 0 \bmod 3$.

If this is so, then $c=0$ and

$$
\begin{aligned}
m & =m_{(0, d)} \geq \sum_{p \in I} m_{(3 p, 3 p+3)} \\
& \geq 21|I|=21\left(\frac{d-3}{3}+1\right)=7 d .
\end{aligned}
$$

Making $d$ subject of the formula, we get $d \leq(m / 7)$.

Case 2. $d \equiv 1 \bmod 3$.

If this is so, then $c=1$ and

$$
\begin{aligned}
m & =m_{(0, d)} \geq \sum_{p \in I} m_{(3 p, 3 p+3)}+m_{(d-1, d)} \\
& \geq 21|I|+5=21\left(\frac{d-1-3}{3}+1\right)+5=7 d-2 .
\end{aligned}
$$

Making $d$ subject of the formula, we get $d \leq((m+2) / 7)$.

Case 3. $d \equiv 2 \bmod 3$.

If this is so, then $c=2$ and

$$
\begin{aligned}
m & =m_{(0, d)} \geq \sum_{p \in I} m_{(3 p, 3 p+3)}+m_{(d-2, d-1)}+m_{(d-1, d)} \\
& \geq 21|I|+5+5=21\left(\frac{d-2-3}{3}+1\right)+10=7 d-4 .
\end{aligned}
$$

Making $d$ subject of the formula, we get $d \leq((m+4) / 7)$. To show that this bound is tight, consider the extremal graph given in Theorem 6 . Observe that whenever $d \equiv 0 \bmod 3$, $\operatorname{diam}\left(P_{d}\left(\pi_{0}, \pi_{1}, \ldots, \pi_{d}\right)\right)=(m / 7)$.

\section{Conclusion}

In this paper, we provided tight upper bounds for bipartite graphs with respect to order and with respect to size for any value of $\lambda$. We also provided tight upper bounds for trianglefree graphs with respect to order for any value of $\lambda$. Also, for triangle-free graphs, with respect to size, we provided tight upper bounds for any value of $\lambda$ where $\lambda \neq 5$. For $\lambda=5$ with regard to the size of triangle-free graphs, we provide the following conjecture.

Conjecture 1. Let $G$ be a 5-edge-connected triangle-free graph of size $m$. Then,

$$
d=\operatorname{diam}(G) \leq \frac{m+4}{7}
$$

Further, if bound Conjecture 1 is correct, it would also be asymptotically tight. This is so since this bound almost matches the diameter of the extremal graph in Theorem 7 , which is also 5-edge-connected and triangle free, except for a small constant.

\section{Data Availability}

No data were used to support this study.

\section{Disclosure}

The results in this paper are part of the first author's MPhilSc thesis. 


\section{Conflicts of Interest}

The authors declare that they have no conflicts of interest.

\section{References}

[1] M. Akram, m-Polar Fuzzy Graphs, Studies in Fuzziness and Soft Computing, Springer, Berlin, Germany, 2019.

[2] F. R. K. Chung, "Diameters of graphs: old problems and new results," Congressus Numerentium, vol. 60, pp. 295-317, 1987.

[3] G. Chartrand and O. R. Oellermann, Applied and Algorithmic Graph Theory, McGraw-Hill, New York, NY, USA, 1993.

[4] P. Erdős, J. Pach, R. Pollack, and Z. Tuza, "Radius, diameter and minimum degree," Journal of Combinatorial Theory B, vol. 47, pp. 73-79, 1989.

[5] S. Mukwembi, Bounds on distances in graphs, Ph.D. thesis, University of KwaZulu-Natal, Durban, South Africa, 2007.

[6] P. Dankelmann, G. Dlamini, and H. C. Swart, Upper bounds on distance measures in $K_{2, l}$-free graphs.

[7] P. Dankelmann, G. Dlamini, and H. C. Swart, "Upper bounds on distance measures in $K_{3,3}$-free graphs," Utilitas Mathematica, vol. 67, pp. 205-222, 2005.

[8] S. Mukwembi, "The radius triangle-free graphs with prescribed edge-connectivity," Utilitas Mathematica, vol. 77, pp. 135-144, 2008.

[9] P. Dankelmann and S. Mukwembi, "The distance concept and distance in graphs," Kragujevac, vol. 12, pp. 3-48, 2012. 